\title{
Frontières
}

\section{L'exposition traditionnelle des défunts à Lévis (1920-1960)}

\section{Caroline Guay}

Volume 15, numéro 1, automne 2002

Délires urbains, dangers de mort

URI : https://id.erudit.org/iderudit/1073906ar

DOI : https://doi.org/10.7202/1073906ar

Aller au sommaire du numéro

Éditeur(s)

Université du Québec à Montréal

ISSN

1180-3479 (imprimé)

1916-0976 (numérique)

Découvrir la revue

Citer cet article

Guay, C. (2002). L’exposition traditionnelle des défunts à Lévis (1920-1960).

Frontières, 15(1), 47-51. https://doi.org/10.7202/1073906ar

\section{Résumé de l'article}

L'exposition des défunts à la maison était pratique courante dans les familles québécoises au début $\mathrm{du} \mathrm{XX}^{\mathrm{e}}$ siècle. Les témoignages de personnes interrogées dans le cadre d'une enquête orale nous décrivent comment se passaient les choses à Lévis. d'utilisation que vous pouvez consulter en ligne.

https://apropos.erudit.org/fr/usagers/politique-dutilisation/ 


\section{Résumé}

L'exposition des défunts à la maison était pratique courante dans les familles québécoises au début $\mathrm{du} X \mathrm{X}^{\mathrm{e}}$ siècle. Les témoignages de personnes interrogées dans le cadre d'une enquête orale nous décrivent comment se passaient les choses à Lévis.

Mots clés : rite - exposition - Lévis

\section{Abstract}

At the beginning of the twentieth century, the deceased's being laid out at home was current practice among Quebecois families. Accounts from people interviewed in an oral survey describe to us how things would happen in Lévis.

Key words : rite - wake - exposition Lévis

\section{L'EXPOSITION TRADITIONNELLE DES DÉFUNTS À LÉVIS (1920-1960)}

\begin{abstract}
Caroline Guay,
étudiante au doctorat en sciences des religions, UQÀM.
\end{abstract}

Qu'elle soit qualifiée de traditionnelle ou de moderne, d'archaïque ou de contemporaine, aucune collectivité humaine n'a semble-t-il cédé les siens à la "grande faucheuse » sans marquer l'événement de différents rites, prodiguant à ses cadavres plus ou moins de soins et leur offrant, sous une forme plus ou moins élaborée, une sépulture, ce qu'Edgar Morin identifie comme une « donnée première, fondamentale, universelle de la mort» (Morin, 1970, p. 32). Il est possible de considérer que le cadavre devient ainsi le " point zéro » du rituel funéraire, pour reprendre l'expression de LouisVincent Thomas ; c'est du moins la position qui est adoptée ici pour décrire le rituel de l'exposition du défunt dans la ville de Lévis, dans les années 1920 à 1960.

Dans le Québec traditionnel, les rites funéraires étaient régis, voire strictement contrôlés, par l'idéologie catholique à l'époque très influente et, comme le signale le narrateur du film Miroir de la vie et de la mort, Michel Garneau: "Au temps où la religion était responsable de la mort, elle avait mis en place une organisation du deuil, qui débouchait sur l'optimisme de la foi...» (Brault, 1985).

Cet article s'inspire d'une recherche qui avait pour but d'illustrer la mutation du rite de l'exposition du défunt au Québec (Guay, 2002). Lillustration de la mort traditionnelle présentée ici est directement issue d'une enquête orale, où les souvenirs d'une quinzaine de personnes ont été mis à profit dans le but de documenter ce rite.
Le sociologue français Claude Rivière a élaboré un cadre d'analyse des rites et je m'en inspire pour la présentation des données recueillies lors de l'enquête orale ${ }^{1}$. Le rite est envisagé comme une réalité complexe, « composée d'éléments jamais tout à fait ajustés » (Rivière, 1997, p. 103) et perméable à maintes influences. Selon Rivière, un rite peut être soumis à une segmentation en cinq temps, conservée pour la suite de cet article : « Méthodologiquement, tout rite, aussi bien profane que religieux, peut être appréhendé comme structure d'actions séquentielles, de rôles théâtralisés, de moyens réels et symboliques, de communication par système codé » (Rivière, 1995, p. 78). Tout comme Louis-Vincent Thomas, Rivière pose comme postulat principal que le rite trouve sa finalité en lui-même et, pour reprendre les termes de Zakaria Jéridi, «n'a d'autre projet que son propre accomplissement » (Jéridi, 1996, p. 161). Il est un aspect que Rivière n'élabore guère : l'espace. J'en ferai ici une catégorie additionnelle, en plus des structures d'actions séquentielles, des rôles théâtralisés, des moyens et des communications.

Reprenons un peu plus longuement chacune de ces structures. D'abord, la structure temporelle de l'action se retrouve dans l'enchaînement des actions que posent des individus placés en situation de conflit émotionnel, lequel peut être déclenché par un événement traumatisant, comme le décès d'un membre de la communauté dans le cas présent. Chacune de ces actions devient, selon Rivière, un "épisode homogène " (Rivière, 1997, p. 104) qui, lorsque rattaché à d'autres épisodes, forme l'ensemble du 
rite. La structure des rôles, quant à elle, veut expliciter les différents états qu'occupent les acteurs dans le déroulement du rite et leurs « conduites stéréotypées » (Rivière, 1997, p. 106), bref, les situations où les acteurs sociaux jouent leur rôle dans une conjoncture rituelle donnée. Toutes ces conduites et ces manières d'être se trouvent en quelque sorte mises en scène dans ce que Rivière nomme un drame institué. Pour que le rite atteigne l'efficacité sociale, la structure des moyens regroupe divers éléments mis en place plus ou moins consciemment, "afin d'entrer en contact avec le numineux ${ }^{2}$ ou d'acquérir paix et certitude » (Rivière, 1997, p. 110). Rivière identifie entre autres comme moyens potentiels le lieu sanctuarisé, qui circonscrit le déroulement du rite à un lieu particulier, où se déroule l'ensemble ou une partie du rite; le temps défini, qui lui délimite un temps précis d'une part pour l'emplacement du rite dans l'horaire de la collectivité et d'autre part pour la durée de l'ensemble du rite lui-même; les objets, moyens très concrets devant soutenir l'évolution du rite ; finalement, les gestes, paroles et attitudes ponctuant les diverses étapes rituelles. Les paroles sont envisagées ici dans le sens de "prière, chant, slogan, discours » (Rivière, 1997, p. 110), et ne sont pas incluses à proprement parler par Rivière dans la structure des communications. Celle-ci illustre plutôt le flux des paroles circulant entre les émetteurs et les récepteurs, la «transmission de messages chargés d'une efficacité mystique » (Rivière, 1997, p. 111). Le sociologue ajoute encore que ces messages prennent place au sein de «systèmes de signification à partir de codes culturellement définis » (Rivière, 1995, p. 50). Il s'agit en fait de faire sens et d'atteindre par le rite une satisfaction symbolique à cette perte, ce vide provoqué par l'absence subite d'un membre de la communauté.

\section{LES ACTIONS SÉQUENTIELLES}

Dans son dossier sur la mort au Québec, Réal Brisson a très bien énoncé la réaction sociale autrefois provoquée par la mort d'un membre du groupe :

En milieu rural (et jusqu'à une époque encore récente dans les paroisses urbaines), parents et voisins s'engagent dès le début du processus funéraire mis en branle par l'annonce d'un décès. La première tâche consiste à faire la toilette du défunt. S'il doit y avoir embaumement, on fait appel au spécialiste du village, le croque-mort en l'occurrence, ou à un membre de la famille qui est chargé de la préparation du cadavre pour son exposition «sur les planches », c'est-à-dire sur un chevalet recouvert d'un drap blanc et placé dans le salon du logis. (Brisson, 1988, p. 23)

L'annonce formelle du décès se faisait à l'église, le dimanche suivant : le prêtre informait ses paroissiens de la disparition d'un des leurs et indiquait la date des funérailles. Par ailleurs, comme le remarque très justement une informatrice (Simone) : "dans une paroisse, les nouvelles se communiquent assez vite, hein! » Marthe précise :

Moi, je me rappelle en 43, j'ai perdu un frère, j'étais toute jeune... mon père était allé à l'église pour l'annoncer... on sonnait les cloches, y'étaient tout organisés, mais je me rappelle d'une chose, c'est que quand on annonçait le décès le dimanche matin à la messe, c'était à la grand messe, la famille ne devait pas être présente à l'église quand on annonçait le décès d'un membre... (Marthe)

Force est donc de constater que ce moment était vécu difficilement par les plus proches membres de la famille, et c'est probablement la raison pour laquelle ils étaient absents lors de l'annonce officielle à l'église : "Y'avait trop d'émotions pis ils retenaient ça... parce que moi quand papa est mort en 32, maman est pas allée à l'église...» (Rolande). Précisons que l'annonce du décès s'effectuait parallèlement aux nombreuses mesures mises en place tant pour préparer l'exposition du cadavre que pour recevoir les visiteurs venus lui rendre un dernier hommage.

À la lumière des informations obtenues, on distingue deux façons de faire quant à la préparation du cadavre, l'une n'impliquant pas l'intervention d'un spécialiste et l'autre où le cadavre était confié à un embaumeur. Dans le premier cas, le corps était laissé aux mains d'une ou plusieurs femmes, la plupart du temps des voisines:

Ils les embaumaient pas dans ce temps-là... chez nous, à ce moment-là, des personnes embaumées par un embaumeur, tu n'en voyais pas, y n'en venaient pas, comprenez-vous... à part ça y'avait pas d'entrepreneur pour le service non plus, tout se faisait... ça c'est aux alentours de 1928, y'a coulé de l'eau dans la rivière depuis ce temps-là, hein! (rires) Et pis ensuite, les femmes, c'est eux autres qui faisaient l'embaumement, les femmes voisines assez souvent, elles faisaient la toilette pis l'embaumement y'en avait pas, mais elles préparaient le défunt pour le mettre dans sa tombe... (Simone)
On le voit donc, ici, il n'y avait pas d'intervention interne sur le cadavre. Il s'agissait de le laver sommairement, de le coiffer et de le vêtir de ses plus beaux atours, bref le rendre le plus présentable possible pour l'exposition. Dans le deuxième cas, il $\mathrm{y}$ avait contribution d'une entreprise de pompes funèbres qui pouvait soit préparer le cadavre directement sur les lieux du décès, soit le transporter dans ses locaux pour ensuite le ramener à la maison. Quand l'embaumement avait lieu à la maison, il semble que l'opération suscitait bien des interrogations : «Mon frère a été embaumé chez nous (1943, sur la Côte-de-Beaupré), je me souviens de ça... j'ai trouvé ça épouvantable ! Je le voyais aller aux toilettes avec des bassins pis revenir... c'est toujours resté dans ma tête, c'est quoi qu'y faisait là ?» (Marthe). On peut comprendre la peur que pouvait éventuellement inspirer la manipulation du cadavre, relatée ici par un embaumeur à la retraite :

Le mort, naturellement, il était dans une chambre à coucher... on avait une table pliante, c'était plié en deux, ça, comme une valise, là... ça faisait une table, on mettait le mort dessus, pis là on l'embaumait... c'est un produit chimique, ça gèle les chairs, faut savoir comment travailler avec... le sang c'étaient des pots en verre, ça y'avait un couvert dessus, le sang coulait dans ça... j'avais une casserole en stainless, là, je lui embarquais les épaules dans ça, pour le saigner, pis le sang coulait là-dedans, pis ça coulait dans les pots, avec des hoses, on avait des coffres, on amenait ça au bureau (locaux de l'entreprise de pompes funèbres) pis on jetait ça, nous autres ça allait dans les égouts... fallait enlever ce qui était brisé, pis refaire ça en neuf, si c'était une joue, un œil, les lèvres... on mettait, le temps qu'on faisait l'embaumage, on mettait une crème de base, une crème blanche, pis quand on avait fini de l'embaumer, pour mettre les cosmétiques, on essuyait un peu le corps, là... on avait des couleurs pour maquiller le corps, on mettait du rouge à lèvres... (Hugues)

Quand le corps était sorti de la maison pour l'embaumement, c'était aussi l'entrepreneur qui s'occupait de gérer le déplacement du cadavre tant à l'aller qu'au retour des locaux de l'entreprise, comme le raconte une informatrice à propos de la mort de son grand-père en 1944 :

Ce que je me souviens, c'est que lorsque l'entrepreneur des pompes funèbres est entré dans la chambre... je 
peux pas savoir comment ça se fait que j'étais là, en tout cas, fallait que je sois là pour vous raconter ça aujourd'hui, hein! (rires) Là, je me souviens très bien que l'entrepreneur de pompes funèbres est arrivé, il l'avait enveloppé dans un drap, et puis il l'avait mis dans un grand panier de jonc, ça je me souviendrai de ça, ça m'avait frappée, alors ils sont partis avec... (Francine)
Ainsi, nous constatons que le cadavre n'était jamais exposé sans un minimum de soins, que ceux-ci soient prodigués par une voisine ou un embaumeur.

Autrefois, l'exposition du cadavre avait lieu dans la maison même où résidait le défunt de son vivant, et durait en moyenne trois jours (pas plus d'une journée cependant pour les jeunes enfants), tel qu'en témoigne une informatrice: «On les gardait deux à trois jours dans la maison, jour et nuit, là... les nuits, dans l'temps, c'est nous autres qui les gardaient...» (Simone). Notons que les informateurs insistent tous sur le fait que le cadavre n'était jamais laissé seul. Lorsque la nuit arrivait, il semble que l'ambiance changeait quelque peu de nature. L'atmosphère devenait en quelque sorte moins lourde, ce que certains évoquent, non sans un certain malaise, sans trop donner de détails :

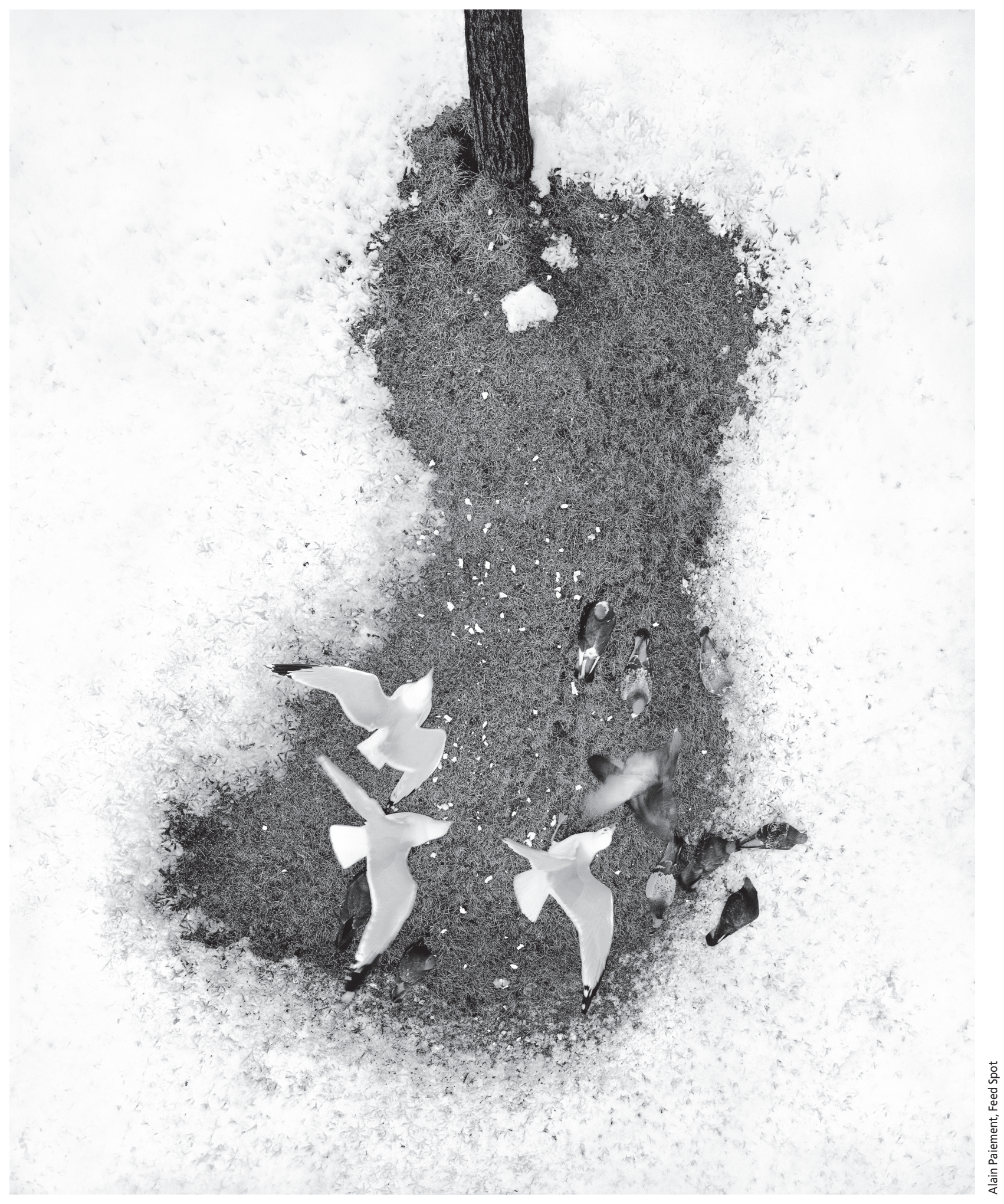


Y'avait une affaire, la nuit, quand c'étaient des jeunesses, ça se contait des histoires ma fille, pis ça riait plus que... mais la plupart du temps le mari était allé se coucher pour voir le monde qui venait dans le jour, tu comprends... mais c'était rien qu'entre jeunes, la peine était moins profonde, ça se contait des histoires, pis ça j'en ai entendu parler, pis pas rien qu'à une place! (Thérèse)

Le défunt n'était pas veillé exclusivement par ses parents et amis. En effet, Simone relate un fait particulièrement intéressant :

Dans les villes, ce qui se faisait, j'avais une sœur qui travaillait justement ici, à Lévis, et puis où elle travaillait, le monsieur était décédé, alors pour la nuit, eux autres, ils engageaient des religieuses, pour garder la nuit, c'était bien hein... vers huit heures, neuf heures, le soir, les religieuses venaient, pis ça allait jusqu'au matin, les religieuses gardaient, deux religieuses qui gardaient le défunt... (Simone)

\section{LES RÔLES THÉÂTRALISÉS}

C'est à la famille immédiate qu'incombe la plus grande part de responsabilité dans le déroulement du rite traditionnel. Non seulement elle doit parfois s'assurer de la préparation du cadavre elle-même, elle doit aussi veiller à la disposition des lieux de manière à créer un environnement propice à l'accueil des visiteurs venus témoigner leur respect au défunt et offrir sympathies et condoléances à la famille.

Les divers préparatifs relatifs à l'accueil des visiteurs étaient semble-t-il principalement assumés par les femmes, les hommes ayant surtout pour tâche la veille du cadavre, la nuit. En plus de parfois préparer le cadavre, donc, les femmes devaient aussi voir à la bonne marche de la maisonnée, préparant les repas, s'occupant des enfants, en plus d'avoir à combler les besoins ponctuels des visiteurs. La remarque d'une informatrice à cet égard est d'ailleurs évocatrice :

Les femmes avaient de l'ouvrage... parce qu'elles faisaient à manger puis elles recevaient tout le monde...

Probablement, aussi que y'avait d'autres gens qui apportaient de la nourriture... ça, je me souviens pas, mais je voyais ma tante qui était occupée... de temps en temps, elle allait faire un petit tour voir mon grand-père (le défunt exposé, en l'occurrence), mais elle revenait et ça été trois jours de temps... (Francine)
Quant à l'embaumeur, remarquons d'emblée que le terme «croque-mort » n'est pas utilisé par les informateurs pour le désigner, qui lui préfèrent la plupart du temps le terme «entrepreneur». À l'époque traditionnelle, le métier d'embaumeur en était à ses balbutiements. Devenait embaumeur celui qui avait été suffisamment longtemps apprenti d'un confrère plus expérimenté. Ce n'est que dans les années 1950 que l'on retrouve à Lévis des embaumeurs formés dans une école spécialisée. À cette époque, l'une des tâches de l'embaumeur, outre celle de la préparation du cadavre et de son transport si nécessaire, consistait à fournir les éléments décoratifs appropriés pour l'exposition à la maison. Une fois le défunt préparé et le décor mis en place, commençaient à affluer les visiteurs. D'une part, il y avait la famille élargie, puis les voisins et amis, et, d'autre part, les représentants des diverses institutions locales. Parmi eux, notons entre autres la présence du prêtre et du bedeau de la paroisse, du médecin, du notaire, du maire ou encore de religieuses. De plus, si la collectivité comptait parmi elle des membres de diverses organisations sociales, celles-ci se faisaient un devoir de déléguer une partie de leurs effectifs pour les représenter, comme le remarque une informatrice: "Les sœurs, oui, quand y'en avait dans la famille... le prêtre venait toujours dire un chapelet... dans ce temps-là, y'avait la Ligue du SacréCœur, y'avait les dames de Sainte-Anne, la congrégation venait un soir dire un chapelet pour ça...» (Thérèse). On le voit, donc, la communauté entière était mobilisée par le décès d'un de ses membres, et aller visiter la famille affligée par cette perte était considéré comme une obligation morale, parce que «dans les paroisses, là, dans ce tempslà, ça se connaissait toutte, tout le monde de la paroisse se faisait un devoir d'aller voir...»(Andrée).

\section{LES MOYENS RÉELS ET SYMBOLIQUES}

La mise en œuvre du rite de l'exposition du cadavre s'exprime par un certain nombre de moyens symboliques et réels, tels que définis par Rivière : lieu sanctuarisé, temps défini, objets divers, gestes et attitudes, sont autant de moyens de participer à l'efficacité du rite. D'abord, le salon de la maison privée est momentanément transformé, entre autres par les décorations et « l'atmosphère de deuil et de respect» (Béliveau, 1994, p. 56) qui y règne, en lieu sanctuarisé. C'est là que se déroulent la plupart du temps les prières. En dehors de ces périodes cependant, les visiteurs ne s'attardent pas outre mesure dans le salon, lui préférant généralement la cuisine : «Là, les gens arrivaient, ils saluaient mon grand-père (le défunt exposé), lui disaient bonjour et faisaient une prière, pis après ça ils s'en allaient dans la cuisine, ils restaient pas dans le salon... »(Francine). Le temps défini, lui, se limite dans le cas présent à la période d'exposition proprement dite, allant de deux à trois jours complets, jour et nuit, comme mentionné ci-haut. Ensuite, au nombre des objets mis à contribution dans le cadre de ce rite, les objets de couleur noire jouent un rôle de premier plan. C'est par ce moyen très réel que s'exprimaient les marques de deuil entourant un décès :

Le noir domine l'ensemble du cérémonial traditionnel de la mort. À la maison comme à l'église, le code vestimentaire approprié de même que le mobilier et les autres éléments du décor funèbre imposent au deuil ses couleurs sombres. Un crêpe noir accroché à la porte principale de la maison signale une mortalité. (Brisson, 1988, p. 27)

Ce crêpe, d'ailleurs, semblait inspirer une certaine crainte, aux enfants en particulier : «C'était sinistre! Le crêpe à la porte, là... un gros crêpe noir là... quand on était en campagne, y'a quelques soirs qu'on sortait pas... pis on passait dans la rue pis y'avait un crêpe noir, on avait assez peur qu'on traversait la rue pour pas passer devant la maison! » (Aurèle.) C'est aussi de noir que devait se revêtir la famille du défunt, pour une période allant de six mois à un an, selon le lien familial entre le survivant et le défunt. Cette pratique n'a plus cours aujourd'hui, au grand soulagement d'ailleurs d'une informatrice :

Comme porter le deuil un an de temps, le noir, après ça six mois de demideuil, en mauve, violet, pis le gris... m'a dire comme des fois, moi, c'est une affaire que j'étais pas capable, y'en a qui disaient que c'était un sacrifice pour ceux qui étaient partis, mais moi je me dis que ça, là, pantoute... la peine, tu l'exposes pas, tu l'as en dedans de toi, ça, j'étais ben contente quand ça a arrêté, le linge noir... (Thérèse)

Toutefois, dans le cas de bébés et de jeunes enfants, le blanc était de mise pour tous les éléments impliqués dans le rituel, des vêtements de l'enfant au cercueil et au corbillard. Au nombre des objets présents dans le lieu d'exposition, outre le cercueil qui la plupart du temps était fabriqué par un homme de la paroisse, notons la présence d'objets liés au culte catholique, tels que le prie-Dieu, le chapelet, les cierges, le crucifix, dont la disposition ne semblait pas être laissée au hasard, bien qu'il n'existât pas de règles strictes. Finalement, toujours 
au niveau des objets concrets devant soutenir le déroulement du rite, il faut noter l'importance de la nourriture.

C'est que l'acte de manger et de boire ensemble, à l'occasion de la mort, permet de se relier les uns aux autres, de manière presque communielle, et parfois même autorise la participation à l'excès qui est bien le propre des forces de vie. Manger pour se sustenter, soit, mais manger pour signifier que l'effacement des individus ne saurait menacer la collectivité.

(Des Aulniers et Lévy, 1991, p. 5)

L'ingestion de nourriture était à l'époque partie prenante du rituel. En effet, il n'était pas rare qu'une ou plusieurs femmes de la maisonnée s'activent autour du poêle toute la journée, parce que tout ce beau monde devait bien être nourri. Cette responsabilité incombait à la famille qui recevait la visite de gens venus offrir leurs sympathies. En plus des repas servis aux heures habituelles de la journée, il n'était pas rare, voire de mise, d'offrir de la nourriture aux visiteurs qui partaient après une longue soirée de veille ainsi qu'à ceux qui assuraient la garde pour la nuit, la plupart du temps des hommes: «D'abord, le monde pouvait partir vers onze heures, là nous autres on s'occupait de mettre... j'me rappelle de ça quand moman est morte (en 1944), on faisait... on appelait ça un lunch, en tout cas, rien de chaud, mais tout de même...» (Julienne). À propos de ce repas tardif, trois des informateurs ont utilisé le terme « réveillon », et l'expression souligne bien l'aspect exceptionnel d'une telle situation.

Enfin, parmi les «gestes et attitudes» relevés par Rivière comme moyens réels et/ou symboliques destinés à accroître l'effet du rituel, notons la prière. Si seulement une informatrice insiste sur le fait qu'un " chapelet se disait à toutes les heures » (Simone), la plupart des personnes interrogées remarquent qu'il n'y avait pas d'horaire fixe: "De temps en temps on avait le chapelet, là, les prières d'usage... les gens se mettaient à genoux dans la cuisine, c'est parce qu'il y avait plusieurs personnes et que le salon était petit, ils disaient le chapelet...»(Francine).

\section{LA COMMUNICATION PAR SYSTĖME CODÉ}

Les données à cet égard sont rares. Une informatrice remarque que les gens «parlaient de chevaux, des animaux, des foins, parce que c'étaient des cultivateurs...» (Andrée). Il semblerait que l'on «parlait ben de toutes sortes de choses, mais ça parlait pas de mort... »(Julienne). Serait-il juste de conclure que l'absence de paroles au sujet de la mort ou du mort témoignerait sim- plement du tabou entourant ces derniers? Il est difficile de répondre à cette question ; chose certaine, aucun des informateurs n'a cru bon d'élaborer à ce sujet, faisant preuve d'une retenue qui ne peut s'expliquer autrement que par une certaine forme de pudeur.

\section{L'ESPACE}

Le modèle d'analyse proposé par Claude Rivière n'aborde pas spécifiquement l'espace en tant que structure. Pourtant, le rite de l'exposition du cadavre implique à l'évidence une délimitation de territoire. $\mathrm{Ce}$ que l'on pourrait appeler une sacralisation territoriale s'exprime entre autres par les éléments décoratifs qui entourent immédiatement le mort (tentures noires, lumières tamisées, etc.) et par le silence que l'on observe habituellement autour de lui. Si l'un des informateurs remarque que le cercueil était contre le mur, et que le "monde circulait pas alentours..., comme dans les salons d'aujourd'hui » (Thérèse), d'autres mentionnent que le cercueil prenait place en plein milieu du salon, " la tête au mur et les deux cierges de chaque côté » (Andrée). De cette façon, les gens pouvaient se regrouper autour du défunt, en particulier dans les moments de prière. Tous les informateurs ont été unanimes quant à la pièce utilisée pour l'exposition, soit le salon, bien que dans certains cas une autre pièce pût être aménagée. La cuisine, aussi, devenait semble-t-il un lieu de rassemblement, par opposition au lieu de piété qu'était le salon: "Y'avait toujours quelqu'un qui veillait, y'allait le voir, mais par contre ça se passait pas dans le salon, ils traversaient à la cuisine... » (Francine). Ce «ça », cette socialité mouvante, trouvait dans la cuisine tout l'espace nécessaire pour s'exprimer, dirait-on.

Les données rassemblées ici, sans être exhaustives, semblent toutefois bien témoigner de ce que plusieurs informateurs québécois ont appelé "l'ambiance familiale» qui régnait lors de l'exposition traditionnelle des défunts à la maison dans la première moitié du $\mathrm{XX}^{\mathrm{e}}$ siècle. Le but visé était de recueillir une description de ce rite auprès des acteurs eux-mêmes et de mieux connaître leur perception de ce vécu. Ce travail de consignation de la mémoire pourrait être vu comme un hommage aux recherches patientes des folkloristes d'antan qui ont entre autres permis de laisser une trace de comportements humains aujourd'hui révolus.

\section{Bibliographie}

BRAULT, F. (1985). Miroir de la vie et de la mort, Office national du film du Canada, en collab. avec la Société Radio-Canada.

BRISSON, R. (1988). La mort au Québec. Dossier exploratoire, Québec, Université Laval, Rapports et mémoires de recherche du CÉLAT, $n^{\circ} 12$.

DES AULNIERS, L. et J.-J. LÉVY (1991). "Croquons, croquons-la: alimentation, nourriture et mort (première partie) », Frontières, vol. 3, n 3, p. 5-10.

GUAY, C. (2002). L'exposition du défunt à Lévis, des planches au salon funéraire: exploration d'une mutation, Mémoire de maîtrise en sciences des religions, Université du Québec à Montréal.

JÉRIDI, Z. (1996). «Les mirages du sacré... Le rite et la religiosité revisités ", Cahiers internationaux de sociologie, vol. 100, p. 151-161.

MORIN, E. (1970). L'homme et la mort, Paris, Seuil.

OTTO, R. (1995). Le sacré, Paris, Payot et Rivages.

RIVIÈRE, C. (1995). Les rites profanes, Paris, Presses universitaires de France.

RIVIÈRE, C. (1997). "Structure et contrestructure dans les rites profanes", dans M. SEGRÉ (dir.), Mythes, rites, symboles dans la société contemporaine, Paris, L'Harmattan, p. 101-122.

THOMAS, L.-V. (1980). Le cadavre: de la biologie à l'anthropologie, Bruxelles, Éditions Complexe.

\section{Notes}

1. Chaque citation est suivie du prénom fictif de l'informateur.

2. À propos de la notion de numineux, citons Rudolf Otto: "Le sentiment de l'état de créature n'est au contraire qu'un élément subjectif concomitant, un effet; il est pour ainsi dire l'ombre d'un autre sentiment, celui de l'effroi, qui, sans aucun doute, se rapporte d'emblée et directement à un objet existant en dehors du moi. Cet objet, c'est précisément l'objet numineux. Ce n'est que là où l'on éprouve la présence du numen, comme dans le cas d'Abraham, où l'on ressent quelque chose de caractère numineux, où l'âme se tourne d'elle-même vers cet objet, en d'autres termes, ce n'est que par l'effet d'une application de la catégorie du numineux à un objet réel ou présumé, que peut surgir, comme réaction dans la conscience, le sentiment de l'état de créature» (Otto, 1995, p. 25). 